\title{
PERCEPCION DEL RIESGO LABORAL EN TRABAJADORES OPERATIVOS DEL SECTOR METALMECÁNICO
}

\author{
Irlena Ahumada Villafañe ${ }^{1 *}$, Jose Palacio Angulo ${ }^{2}$, Jorge Posada López ${ }^{3}$, Iván Darío Orjuela \\ ${ }^{1,2,3,4}$ Corporación Universitaria Minuto de Dios - UNIMINUTO. Barranquilla, Colombia \\ *Autor de correspondencia: irlena.ahumada@uniminuto.edu.co
}

Recibido Agosto 2019; Aceptado Noviembre 2019

\begin{abstract}
Resúmen - Se diseñó un protocolo para la percepción del riesgo laboral con enfasis preventivo en accidentes y enfermedades laborales para las empresas del sector metalmecánico de la ciudad de Barranquilla, ubicado al norte de Colombia. La investigación es de tipo no experimental, descriptiva y transversal; para este estudio se utilizó, un cuestionario integrado por 74 ítems, divididos en 3 apartados: datos demográficos, conocimiento en prevención de riesgos laborales y la identificación de los factores de riesgos. Para validar el instrumento se utilizó el Alfa Cronbach el cual alcanzó un valor de 0.95. Los principales hallazgos nos indican que, los trabajadores presentan un nivel bajo en prevención de accidentes y enfermedades laborales. Se evidencia falta de intervención del gobierno y pocos recursos de los empleadores para cumplir con la normatividad vigente.
\end{abstract}

Palabras Clave: Accidentalidad empresarial, pymes metalmecanicas, salud laboral, seguridad industrial.

\section{PERCEPTION OF LABOR RISK IN OPERATING WORKERS IN THE METALMECHANICAL SECTOR}

\begin{abstract}
A protocol was designed for the perception of occupational risk with a preventive emphasis on accidents and occupational diseases for companies in the metalworking sector of the city of Barranquilla, located in the north of Colombia. The research is non-experimental, descriptive and transversal; For this study, a questionnaire made up of 74 items was used, divided into 3 sections: demographic data, knowledge of occupational risk prevention and identification of risk factors. To validate the instrument, the Cronbach Alpha was used, which reached a value of 0.95 . The main findings indicate that workers have a low level of prevention of accidents and occupational diseases. There is evidence of a lack of government intervention and few resources from employers to comply with current regulations.
\end{abstract}

Keywords: Business accident, small and medium metalworking company (PYMES), occupational health, industrial safety.

IPSA SCIENTIA - Vol. 4 № 1 - 2019 


\section{Percepción del riesgo laboral en trabajadores operativos del sector metalmecánico}

\section{Introducción}

Los accidentes de trabajo y las enfermedades laborales representan un problema para un Estado y principalmente para las empresas, su origen se debe a los actos y condiciones inseguras (Zamorano et al., 2009). En efecto, para una adecuada gestión en prevención de riesgos laborales es necesario el cumplimiento de los requisitos legales; es el camino para que dicha gestión comience a calificarse como eficaz (Bedoya, 2015; Ahumada et al., 2016).

Siendo así, la legislación en materia de prevención en los últimos años en Colombia se ha vuelto más exigente con la reciente expedición de la Resolución 1111 de marzo 27 de 2017 al momento de salir del ordenamiento jurídico colombiano; define las fases mínimas para la adecuación y transición del Sistema de Gestión de la Seguridad y Salud en el Trabajo. Pero, no ha sido suficiente para eliminar los riesgos en su totalidad (Bedoya, 2015; Carrasco et al., 2009; Herrán et al., 2016).

En realidad, la industria metalmecánica de las pequeñas organizaciones sigue siendo el motor de la economía de Colombia; muchas de ellas se encuentran en la informalidad (Ochoa \& Ordóñez, 2004). Aún asi, una de las causas del problema y que cuestiona el presente estudio; es el abandono por parte del Ministerio de Trabajo, por falta de inspecciones. Más aun, falta de apoyo de las administradoras de riesgos a las pequeñas empresas que tienen sus trabajadores afiliados al sistema general de riesgos laborales SGRL (García, 2008; Marrugo, 2017). Ahora, el panorama es poco alentador: violación de los procedimientos, negligencia, incomprensión de las normas, supervisión deficiente, falta de planeación planeadas, entre otras. En efecto, esas empresas que prestan sus servicios e insumos; al no tener conocimiento en dicha temática; representa un problema para los colaboradores, familiares, la imagen del negocio, para la ciudad y para el mismo país (Briceño, 2003).

Entre los antecedentes tenemos a Bedoya (2015) realizo una investigación sobre el comportamiento de la accidentalidad en una empresa metalmecánica de Cartagena, Colombia. Se encontró un total de 36 accidentes laborales, en el cual las prevalencias fueron entre otras: cargos afectados ayudante de planta con $33 \%$, aprendiz $22 \%, 75 \%$ de los afectados son de la empresa, mientras el $25 \%$ restante pertenecen a contratistas. (pág. 93). Cuando existe la intención de prevenir riesgos laborales en una población trabajadora es necesario la identificación de los factores de riesgos a los que están expuestos los asalariados. "Se considera un conjunto de causas originarias de accidentes y enfermedades de origen laboral” (Henao, 2014; Ormeño, 2019; Jaimes, 2018).

El sector metalmecánico es uno de los sectores industriales de mayor dinamismo en la historia económica de Colombia (Buchelli \& Marín, 2012). La fase embrionaria de este sector se remonta a principios del siglo XIX, en el cual se destacan una serie de propulsores que describen su evolución inicial (Trujillo \& Iglesia, 2012). El desarrollo histórico de la industria corresponde a una diversidad de actividades que integran el sector metalmecánico; van a estar presentes en un número considerado de puestos de trabajo.

"Los procesos metalmecánico transforman los metales ferrosos y no ferrosos en piezas mediantes procesos mecánicos, con o sin el arranque de virutas, cambiando su forma geométrica, para posteriormente realizar un acabado de la superficie de las piezas" (Velosa \& Sánchez, 2012). Del mismo modo, las pequeñas

IPSA SCIENTIA - Vol. 4 № 1 - 2019 
empresas generan empleo y contribuyen a la dimensión del desempleo en Colombia. "cabe señalar que la participación de las PYMES en la economía nacional se ha caracterizado por jalonar su desarrollo y convertirse en grandes generadoras de empleo" (López et al., 2016).

Han promulgado en Colombia leyes que le han dado mayor apoyo y promoción a las pequeñas empresas según sus activos totales y el número de empleados vinculados a dichas organizaciones según la Ley 905 de 2004 (Congreso de la república, 2004). En la Tabla 1 se muestra la clasificación de las PYMES en Colombia.

Tabla 1. Clasificación de PYMES en Colombia

\begin{tabular}{|l|l|l|}
\hline Tipo de empresa & Planta de personal & \multicolumn{1}{|c|}{$\begin{array}{c}\text { Activos totales en } \\
\text { salarios mínimos mensuales }\end{array}$} \\
\hline Pequeñas & $11-50$ & $501-5.000$ \\
\hline Medianas & $51-200$ & $5.001-15.000$ \\
\hline Microempresa & Hasta 10 & Inferior a 500 \\
\hline
\end{tabular}

Fuente: (Montoya et al., 2010). Adaptación Propia

Siendo así, para que haya un accidente de trabajo en una organización este se debe originar por alguna causa para que se pueda clasificar como accidente. Revisemos lo que dicen González et al. (2016) cuando afirma lo siguiente: "Los accidentes laborales pueden generarse por causas inmediatas o básicas: las inmediatas son las que producen el accidente de manera directa y están conformadas por actos inseguros (comportamientos inadecuados de los trabajadores) y condiciones inseguras (Instalaciones, equipos en mal estado y ponen en riesgo de sufrir un accidente a los trabajadores)".

Sin embargo, el concepto de accidente de trabajo en el continente Europeo se encuentra en el RDL 1/1994 "artículo 115: toda lesión corporal que el trabajador sufra con ocasión o por consecuencia del trabajo que ejecuta por cuenta ajena" (Henao, et al., 2015). Además, las enfermedades laborales también hacen parte de la materia de riesgos laborales. "Dicha palabra significa no sólo ausencia de toda enfermedad, incluidas las lesiones, sino, el estado de bienestar físico, psíquico y social, lo que suele llevar a connotaciones casi exclusivamente sanitarias" ya que la salud de una persona puede verse afectada por un suceso repentino dentro y/o fuera de la organización. (Morelos \& Fontalvo, 2013).

Asimismo, lo expresa Collado (2008) "La salud puede perderse por un accidente de trabajo o por una enfermedad adquirida a consecuencia del propio trabajo. En cualquier caso, atendiendo al origen de las causas que dan lugar a la patología del trabajo" (pág.95). Andrade \& Gómez (2013) afirman: "La salud laboral surge como respuesta al conflicto entre salud y condiciones de trabajo, y se ocupa de la vigilancia e intervención sobre las condiciones de trabajo y la salud de los trabajadores" (pág. 11). Cuidando las condiciones de trabajo se evitaran los riegos laborales en los trabajadores. Por otra parte, un autor afirma que las personas con pocos recursos económicos y los trabajadores de menor cualificación son los que enferman rápidamente antes que el rico y los ejecutivos (Cárcoba, 1996).

IPSA SCIENTIA - Vol. 4 № 1 - 2019 


\section{Percepción del riesgo laboral en trabajadores operativos del sector metalmecánico}

Las características de las enfermedades laborales en Colombia se clasifican según lo establecido en la Tabla 2.

Tabla 2. Características de las enfermedades laborales

\begin{tabular}{|c|l|}
\hline Ítem & \multicolumn{1}{c|}{ Características } \\
\hline 1 & $\begin{array}{l}\text { Producida por un agente especifico con relación } \\
\text { directa con la afección y el oficio. }\end{array}$ \\
\hline 2 & Tiempo de exposición \\
\hline 3 & Aparición crónica \\
\hline 4 & En la mayoría de los casos es previsibles \\
\hline 5 & $\begin{array}{l}\text { Ejercicio de una profesión, oficio o trabajo } \\
\text { determinado }\end{array}$ \\
\hline 6 & Alteración del estado normal de salud \\
\hline 7 & $\begin{array}{l}\text { Incapacidad gradual causada por la actividad } \\
\text { laboral }\end{array}$ \\
\hline
\end{tabular}

Fuente: (Henao, et al., 2015). Adaptación propia.

Por otra parte, son importante las actividades de Medicina Preventiva y del Trabajo, porque tiene como propósito observar atentamente los problemas de salud de los trabajadores. Buendía, et al., (2006) afirman: "la correcta gestión y protección de la Salud de los trabajadores tiene el desarrollo de guías o procedimientos de vigilancia de la Salud" así como también organizar e implantar un servicio oportuno de primeros auxilios. Por lo tanto, la Medicina Preventiva y del Trabajo tiene funciones a desarrollar establecidos en la normatividad. Siendo así, Moreno (2006) afirma lo siguiente: "Estudio de las patologías que se produzcan entre los trabajadores para identificar cualquier potencial relación entre las causas de la enfermedad y los riesgos para la Salud que puedan producirse a resultas de la realización del trabajo".

Los empresarios muchas veces se limitan a la compra de los EPP, pero no se preocupan porque los trabajadores los identifiquen, conozcan su importancia frente a la protección que ellos brindan y sobre todo, la forma como deben guardarse (Jiménez, 2005).

No solamente es suministrar sino también capacitarlos para el buen uso de los equipos e implementos de trabajo. Un ade las áreas de problemas de salud laboral es la auditiva ya que hay varias razones por las que los protectores auditivos fallan para prevenir la pérdida auditiva; dentro de las cuales se encuentran: incomodidad, interferencia para escuchar conversaciones y señales de advertencia, uso incorrecto con otro equipo de seguridad, deterioro y abuso" (Guerrero, et al., 2005).

Se entiende que una empresa con sus trabajadores sanos y seguros es sinónimo de compañías productivas, asi como lo dice: Matabanchoy (2012) "la salud, seguridad y bienestar de los trabajadores son fundamentales para las empresas en términos de productividad, sostenibilidad y competitividad, para el contexto personal y familiar de los trabajadores y para la economía en general" (pág. 88). Por lo tanto, aquellas condiciones de trabajo afectan la satisfacción laboral que percibe el trabajador por factores de ausencia de autonomía, contenido del trabajo, violencia laboral, discriminación, salarios, entre otros (Buelvas, et al., 2013). Por último, Pérez (2011) asegura lo siguiente: "En Colombia, dos de cada tres

IPSA SCIENTIA - Vol. 4 № 1 - 2019 
trabajadores refieren estar expuestos a factores psicosociales durante la jornada laboral completa" (pág. 67). Lo anterior, pertenece al riesgo psicosocial generador de enfermedad laboral.

\section{Materiales y Métodos}

El diseño metodológico corresponde a una investigación de tipo transversal con un enfoque cuantitativo con carácter descriptivo. Esto, se debe a que centró su atención en las pequeñas empresas metalmecánicas de Barranquilla. Combino el método Análisis-Síntesis, puesto que inicialmente se consideran cada una de las partes que conforman la realidad existente.

\section{Población y muestra}

La muestra se determinó por consenso ya que incluye a la población de pequeñas empresas del sector metalmecánico registrada en la cámara de comercio de Barranquilla, con el fin de garantizar la confiabilidad de los resultados de la investigación. El muestreo fue no probabilístico de tipo aleatorio simple. Siendo asi, el error de estimación fue de $0.5 \%$ y el nivel de confianza del $95 \%$. Por lo tanto, el tamaño de la muestra fue de un total de 32 empresas.

\section{Instrumentos}

Para la recolección de datos se utilizó un cuestionario para evaluar los conocimientos en prevención de riesgos laborales y la identificación de los factores de riesgos. Se caracteriza por tener 74 ítems que se agrupan en 3 dimensiones; está dividido en 2 secciones: la primera su escala es tipo Likert, con 5 opciones de respuesta (1. Totalmente de acuerdo, 2. Endesacuerdo, 3. Parcialmente de acuerdo, 4. De acuerdo, 5. Totalmente de desacuerdo). La segunda sección contiene las características demográficas relativas a la edad y género. Aplicándose de manera individual y confidencial, el cuestionario se aplicó a los trabajadores dentro de las instalaciones de las empresas en horario de trabajo. Dicha recolección de datos se llevó a cabo en el mes de octubre de 2017.

La confiabilidad del instrumento (Alfa de Cronbach 0.851) (Oviedo \& Arias, 2005) es considerable aceptable, quien indica la siguiente interpretación de los índices de confiabilidad "coeficiente de confiabilidad de alrededor de 0.95 se considera excelente", ya que cuando se obtiene valores de 0.80 son considerados como muy bueno, valores alrededor de 0.70 , como adecuados; aquellos menores de 0.50 indican que al menos la mitad de la varianza observada puede ser debida a error al azar, medidas tan poco confiables deberían ser evitadas.

\section{Análisis de datos}

El estudio estadístico fue realizado mediante el Statistical package for the social sciences (SPSS) version 17 para Windows. Se llevaron a cabo análisis estadísticos descriptivo, paramétricos y no paramétricos

IPSA SCIENTIA - Vol. 4 № 1 - 2019 
mediante correlaciones de Pearson, chi cuadrado $\left(\mathrm{x}^{2}\right)$ y coeficiente de contingencia (Hernández et al., 2017).

\section{Resultados}

Calculando las frecuencias de las características demográficas, se encontró que el 84,4\% lo ocupa la fuerza masculina de la muestra estudiada, mientras el 15,6\% pertenece al género femenino. Por otra parte, se cuestionó a los trabajadores sobre la deficiencia en las actividades preventivas de seguridad y salud en el trabajo de su empresa. En la pregunta, ¿Su empresa no cuenta con un área de seguridad y salud en el trabajo? el 100,0\% de los encuestados expusieron que están de acuerdo; ninguna de las pequeñas empresas estudiadas no cuentan con dicha área administrativa y operativa.

Ahora, un ítem pidió a los encuestados seleccionar una opción de respuesta con la siguiente pregunta: ¿En su empresa no se realizan actividades de promoción y prevención por parte de la Administradora de Riesgo? (Tabla 3).

Tabla 3. Actividades de promoción y prevención por parte de la Administradora de Riesgo de su empresa

\begin{tabular}{|l|c|c|c|c|}
\hline Caracterízaciones & Frecuencia & Porcentaje & Porcentaje válido & Porcentaje acumulado \\
\hline Totalmente en desacuerdo & 5 & 15.6 & 15.6 & 15.6 \\
\hline Totalmente de acuerdo & 27 & 84.4 & 84.4 & 100 \\
\hline Total & 32 & 100 & 100 & \\
\hline
\end{tabular}

Fuente: Autor

Se evidencia que la mayoría de los trabajadores $84.4 \%$ eligieron la opción de respuesta indicada para señalar que ninguno de ellos ha recibido por parte de las Administradoras de Riesgos Laborales, actividades encaminadas a la promoción y prevención de riesgos.

Por otro lado, el Ministerio de Trabajo es uno de los entes del gobierno que debe vigilar el cumplimiento legal en materia de seguridad y salud en el trabajo en las empresas públicas y privadas de todo el territorio Nacional. Es por eso que una de las preguntas formuladas cuestiona a los trabajadores, para conocer la participación activa del gobierno como se puede observar en la Tabla 4.

Tabla 4. Inexistencia de capacitaciones en prevención de riesgos laborales por parte del Ministerio de Trabajo.

\begin{tabular}{|l|c|c|c|c|}
\hline Caracterizaciones & Frecuencia & Porcentaje & Porcentaje válido & $\begin{array}{l}\text { Porcentaje } \\
\text { acumulado }\end{array}$ \\
\hline Totalmente en desacuerdo & 6 & 18.8 & 18.8 & 18.8 \\
\hline En desacuerdo & 2 & 6.3 & 6.3 & 25 \\
\hline De acuerdo & 4 & 12.5 & 12.5 & 37.5 \\
\hline Totalmente de acuerdo & 20 & 62.5 & 62.5 & 100 \\
\hline Total & 32 & 100 & 100 & \\
\hline
\end{tabular}

Fuente: Autor

IPSA SCIENTIA - Vol. 4 № 1 - 2019 
El 62,5\% expresaron la poca participación del Ministerio que debe velar por el bienestar de cada uno de los trabajadores de Colombia; lo que puede ocasionar un grave problema en la clase trabajadora colombiana. Aún así, se les planteó un interrogante relacionado con inspectores de trabajo que permitiera complementar la pregunta anterior y que sus resultados se presentan a continuación en el Gráfico 1.

Gráfico 1. Ausencia de inspectores de trabajo de la dirección nacional de riesgos laborales de Colombia

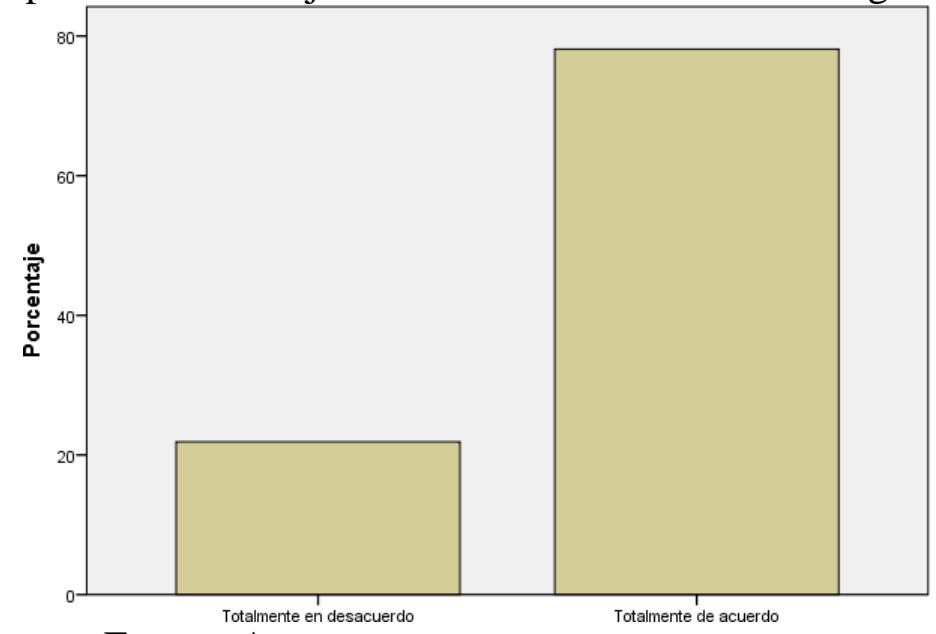

Fuente: Autor

El 78,1\% respondió que están totalmente de acuerdo con la ausencia de inspectores de trabajo, es una situación que genera problema no solamente para la ciudad de Barranquilla sino también para toda Colombia.

Por otro lado, se cuestionó a los participantes, acerca de cada uno de los agentes contaminantes que representan riesgos para los trabajadores de las pequeñas empresas metalmecánica de Barranquilla Colombia. Ahora bien, dichos agentes contaminantes conocidos como los factores de riesgos son los que generan accidentes de trabajo y enfermedad laboral, ellos son: Físicos, químicos, biológicos, psicosocial, biomecánico, de seguridad y fenómenos naturales. Para esto, se preguntó a los participantes, si la temperatura es inadecuada debido a la existencia de fuentes de calor o frío o a la inexistencia de un sistema de climatización apropiado. Estos resultados se muestran en la Tabla 5.

Tabla 5. Temperatura inadecuada. La temperatura es inadecuada debido a la existencia de fuentes de calor o frío o a la inexistencia de un sistema de climatización apropiado.

\begin{tabular}{|l|c|c|c|c|}
\hline Caracterizaciones & Frecuencia & Porcentaje & Porcentaje válido & $\begin{array}{l}\text { Porcentaje } \\
\text { acumulado }\end{array}$ \\
\hline Totalmente en desacuerdo & 10 & 31.3 & 31.3 & 31.3 \\
\hline En desacuerdo en & 2 & 6.3 & 6.3 & 37.5 \\
\hline $\begin{array}{l}\text { Parcialmente } \\
\text { desacuerdo }\end{array}$ & 3 & 9.4 & 9.4 & 46.9 \\
\hline De acuerdo & 7 & 21.9 & 21.9 & 68.8 \\
\hline Totalmente de acuerdo & 10 & 31.3 & 31.3 & 100 \\
\hline Total & 32 & 100 & 100 & \\
\hline
\end{tabular}

Fuente: Autor

IPSA SCIENTIA - Vol. 4 № 1 - 2019 
De la Tabla 5 se observa que el 31,3\% respondieron que la temperatura es inadecuada por la existencia de fuentes de calor. Pero, otro 31,3\% eligió la opción totalmente en desacuerdo, lo que significa que son trabajadores que laboran en condiciones adecuadas.

Adicionalmente en el ambiente laboral de la industria metalmecánica se presentan diferentes riesgos biomecánicos y físicos. En este sentido, al preguntarle a los trabajadores si realizan esfuerzos físicos importantes, bruscos o en posiciones inestable; respondieron lo siguiente que se puede apreciar en el Gráfico 2.

Gráfico 2. Esfuerzo físico importante en su sitio de trabajo.

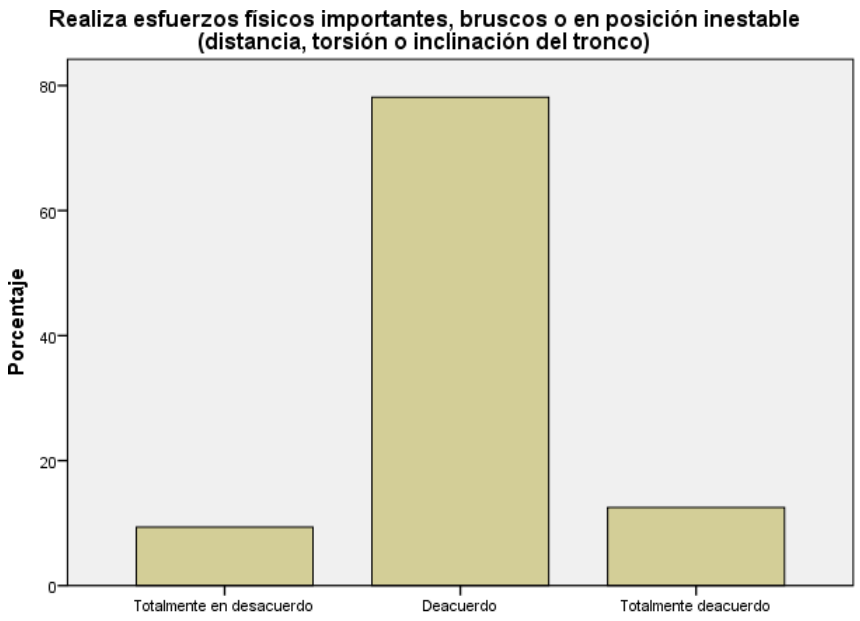

Fuente: Autor

El 75,8\% de los trabajadores encuestados expuso que si realizan esfuerzos fiscos bruscos por la labor que desarrollan constantemente los asalariados.

Por otra parte, los trabajadores del sector metalmecánico están expuesto al material particulado por el polvo metálico, generando enfermedades laborales por el proceso que se realiza con las máquinas de soldar, cortadoras, pulidoras, etc. Al cuestionarles, si están expuesto al polvo metálico, el 93,8\% respondió que sí están expuesto al polvo metálico. En la Tabla 6 se aprecian los resultados.

Tabla 6. Exposición a polvo metálico. ¿Está expuesto usted a polvo metálico?

\begin{tabular}{|l|c|c|c|c|}
\hline Caracterizaciones & Frecuencia & Porcentaje & $\begin{array}{l}\text { Porcentaje } \\
\text { válido }\end{array}$ & $\begin{array}{l}\text { Porcentaje } \\
\text { acumulado }\end{array}$ \\
\hline $\begin{array}{l}\text { Totalmente en } \\
\text { desacuerdo }\end{array}$ & 2 & 6.3 & 6.3 & 6.3 \\
\hline Totalmente de acuerdo & 30 & 93.8 & 93.8 & 100 \\
\hline Total & 32 & 100 & 100 & \\
\hline
\end{tabular}

Fuente: Autor

Sin embargo, al preguntar sobre la existencia de virus, bacterias, hongos, parásitos, exposición a fluidos o excrementos de personas o animales en sus puestos de trabajo; la totalidad de los encuestados respondió que los agentes biológicos no representan ningún riesgo para las industrias metalmecánicas.

IPSA SCIENTIA - Vol. 4 № 1 - 2019 
Al interrogarlos sobre los factores psicosociales, en la pregunta si se sienten ellos discriminados en su entorno laboral, el 12,5\% consideran que si son discriminados. Aún así, en una de las empresas encuestadas algunos trabajadores expresaron que es debido a los despidos injustos que se han presentado.

Por último, se le preguntó a los encuestados si considera que el mantenimiento de los equipos o herramientas es inadecuado resultando los valores que se indican en la Tabla 7.

Tabla 7. Mantenimiento de equipos y herramientas. ¿El mantenimiento de los equipos o herramientas es inadecuado?

\begin{tabular}{|l|c|c|c|c|}
\hline Caracterizaciones & Frecuencia & Porcentaje & Porcentaje válido & $\begin{array}{l}\text { Porcentaje } \\
\text { acumulado }\end{array}$ \\
\hline Totalmente en desacuerdo & 10 & 30.3 & 31.3 & 31.3 \\
\hline En desacuerdo en & 4 & 12.1 & 12.5 & 43.8 \\
\hline $\begin{array}{l}\text { Parcialmente } \\
\text { desacuerdo }\end{array}$ & 1 & 3 & 3.1 & 46.9 \\
\hline De acuerdo & 15 & 45.5 & 46.9 & 93.8 \\
\hline Totalmente de acuerdo & 2 & 6.1 & 6.3 & 100 \\
\hline Total & 32 & 97 & 100 & \\
\hline
\end{tabular}

Fuente: Autor

Respondiendo el 45,5\% de los encuestados que si están de acuerdo con el inadecuado mantenimiento de los equipos y las herramientas manuales, es una de las causas para que se genere un accidente de trabajo. El último cuestionamiento refería a los fenómenos naturales, Barranquilla es una de las ciudades de Colombia que está enfrentando una de las problemáticas sociales como lo son los arroyos producidos por las precipitaciones. Entonces, se les pregunto a los encuestados $i$ se han presentado inundaciones en su empresa? A este cuestionario el 37,5\% de los trabajadores respondió por la opción de acuerdo.

\section{Conclusiones}

Los resultados están demostrando la falta de intervención del gobierno y los pocos recursos de los empleadores para cumplir con la normatividad vigente en riesgos laborales. Por último, esta investigación pretende ser un punto de partida para el desarrollo de nuevos estudios que profundicen el conocimiento de la prevención de los riesgos laborales; mediante el uso de las tecnologías para que exista igualdad y asi bajar la tasa de accidentalidad en Colombia.

\section{Referencias}

- Ahumada Villafañe, I., Escudero Sabogal, I., \& Gutiérrez Jaraba, J. (2016). Normatividad de riesgos laborales en colombia y su impacto en el sector de hidrocarburos. IPSA SCIENTIA: Revista Científica Multidisciplinaria, 1(1), 3142.

- Andrade Jaramillo, V., \& Gómez, I. C. (2013). Salud Laboral Investigaciones realizadas en Colombia. Pensamiento Psicológico, 4(10), 9-25.

IPSA SCIENTIA - Vol. 4 № $1-2019$ 


\section{Percepción del riesgo laboral en trabajadores operativos del sector metalmecánico}

- Bedoya Marrugo, Elías Alberto. (2015). Comportamiento de la accidentalidad en una empresa metalmecánica en Cartagena, Colombia. Nova, 13(24), 93-99.

- Briceño, Leonardo (2003). Prevención de riesgos ocupacionales en empresas colombianas. Revista Ciencias de la Salud, $1(1), 31-44$.

- Buchelli Lozano, G. A., \& Marín Restrepo, J. J. (2012). Estimación de la eficiencia del sectormetalmecánico en colombia: análisis de la frontera estocástica. Cuadernos de Economía, 31(58), 257-286.

- Buelvas, L. M. Í., Oviedo-Trespalacios, O., \& Amaya, C. L. (2013). Condiciones de trabajo que impactan en la vida laboral. Salud Uninorte, 29(3), 542-560.

- Cárcoba, Á. C. (1996). Salud laboral y Ley de prevención de riesgos laborales. Rev Esp Salud Pública, 70(4), 13-57.

- Carrasco Poyatos, María, \& Vaquero Abellán, Manuel, \& Espino Poza, Alberto (2009). Percepción de los riesgos físicos a los que se exponen los profesores de Educación Física de E.S.O. de la provincia de Jaén en su lugar de trabajo. RETOS. Nuevas Tendencias en Educación Física, Deporte y Recreación, (16),115-117.

- Collado, S. L. (2008). Prevención de riesgos laborales: principios y marco normativo. Revista de Dirección y Administración de Empresas, (15), 91-117.

- Congreso de la República (2004). Ley 905 de 2004, por medio de la cual se modifica la Ley 590 de 2000 sobre promoción del desarrollo de la micro, pequeña y mediana empresa colombiana y se dictan otras disposiciones. Diario Oficial No. 45.628, 2 de agosto de 2004.

- García, Alma Clara. (2008). El sistema general de riesgos profesionales vigente en colombia -una visión interna y desde la decisión 584, instrumento andino de seguridad y salud en el trabajo. International Law, 6(13), 216-253.

- González, A., Bonilla, J., Quintero, M., Reyes, C., \& Chavarro, A. (2016). Análisis de las causas y consecuencias de los accidentes laborales ocurridos en dos proyectos de construcción. Revista ingeniería de construcción, 31(1), 05-16.

- Guerrero Pupo, Julio C., Fernández Rojas, Lisset del Rosario, Batista Diaz, Arnaldo, \& Campins Ali, Jandy (2005). Vigilancia ocupacional del trabajador expuesto a alteraciones de la salud inducidas por ruido. Ciencias Holguín, $\mathrm{XI}(1), 1-14$.

- Henao, F. Riesgos físicos: ruido, iluminación y temperatura. ECOE, Ediciones: Colombia, 2014.

- Henao, F., Álvarez, F, Faizal, E \& Valderrama, F. Riesgos Químicos, Biológicos y Bioseguridad. ECOE, Ediciones: Colombia, 2015.

- Hernández de la Rosa, Y., Hernández Moreno, V. J., Batista Hernández, N. E., \& Tejeda Castañeda, E. (2017). ¿ Chi cuadrado o Ji cuadrado?. Medicentro Electrónica, 21(4), 294-295.

- Herrán Villalba, V., Lugo Calderón, E., Puello Martínez, A., \& Ruiz Restrepo, A. (2016). Nivel de conocimiento en riesgos laborales: el caso de trabajadores de servicios generales en una universidad. IPSA SCIENTIA: Revista Científica Multidisciplinaria, 1(1), 43-51.

- Jaimes Morales, J. (2018). Sistema de gestión de seguridad y salud en el trabajo: una revisión desde los planes de emergencia. IPSA SCIENTIA: Revista Científica Multidisciplinaria, 3(1), 23-29.

IPSA SCIENTIA - Vol. 4 № 1 - 2019 


\section{Percepción del riesgo laboral en trabajadores operativos del sector metalmecánico}

- Jiménez Barbosa, I. A. (2005). Afecciones oculares, su relación con factores de riesgo ocupacional y uso de elementos de protección personal en una empresa metalmecánica en Bogotá. Ciencia y Tecnología para la Salud Visual y Ocular, 3(5), 25-30.

- López-Torres, Gabriela Citlalli, Maldonado Guzmán, Gonzalo, Pinzón Castro, Sandra Yesenia, \& García Ramírez, Ricardo. (2016). Colaboración y actividades de innovación en Pymes. Contaduría y administración, 61(3), 568-581.

- Maqueda, J., Buendía, M. J., Gallego, I., Muñoz, C. \& Roel, J. M. (2006). Estudio de evaluación del Protocolo de Vigilancia Sanitaria Específica del Asma Laboral mediante el instrumento AGREE. Med Segur Trab, 52(205), 13-21.

- Marrugo Ligardo, Y. (2017). Health and safety management system in latin america: a review from the hseq integration. IPSA SCIENTIA: Revista Científica Multidisciplinaria, 2(1), 38-45.

- Matabanchoy Tulcán, S. M. (2012). Health at work. Universidad y Salud, 14(1), 87-102.

- Montoya R, A., Montoya R, I., \& Castellanos, O. (2010). Current competitiveness of Colombian SMEs: determining factors and future challenges. Agronomía Colombiana, 28(1), 107-117.

- Morelos, J. \& Fontalvo, T.J. (2013). "Caracterización y análisis del riesgo Laboral en las pequeñas y mediana industria metalmecánica en Cartagena-Colombia.” Rev. soluciones de postgrado EIA, 10 (1), 13-40.

- Moreno, A. (2006). Medicina del Trabajo: Hacia la salud más allá de la vigilancia. Medicina y seguridad del trabajo, 52(205), 3-10.

- Ochoa Valencia, David, \& Ordóñez, Aura. (2004). Informalidad en Colombia: causas, efectos y características de la economía del rebusque. Estudios Gerenciales, 20(90), 105-116.

- Ormeño Bazurto, Luis Alfredo. (2019). Riesgo físico y enfermedades profesionales en trabajadores que operan equipos de vibración en construcciones civiles. Revista San Gregorio, (35), 143-156.

- Oviedo, H. C., \& Arias, A. C. (2005). Aproximación al uso del coeficiente alfa de Cronbach. Revista colombiana de psiquiatría, 34(4), 572-580.

- Perez, E. J. P. (2011). La relación trabajo-estrés laboral en los colombianos. Revista CES salud pública, 2(1), 66-73.

- Trujillo, J.C. \& Iglesia, W. (2012). "Determinante del crecimiento de las Micro, pequeñas y medianas empresas colombianas: el caso del sector metalmecánico.” Semestre Económico, 15 (32), 41-76.

- Velosa García, José Divitt, \& Sánchez Ayala, Luz Marina (2012). Análisis de la capacidad tecnológica en Pymes metalmecánicas: una metodología de evaluación. Revista Escuela de Administración de Negocios, (72),128-147.

- Zamorano González, B., Parra Sierra, V., Peña Cárdenas, F., \& Castillo Muraira, Y. (2009). Conocimiento y actitud en prevención de trabajadores lesionados de una empresa metalmecánica en México. Salud de los Trabajadores, 17(1), 4957. 